ephedrine hydrochloride, $3.6 \mathrm{mg}$ of promethazine hydrochloride, and $9 \mathrm{mg}$ of codeine phosphate. There are no reports in the literature of dependence on phensedyl, although one can only echo the contention of Whitehouse \& Duncan that abuse of substances containing ephedrine occurs unreported in the UK.

Case Report: A 31-year-old single man requested help in stopping drinking 2-3 bottles $(200-300 \mathrm{ml}$ ) of Phensedyl per day. He had been drinking this for 11 years, along with about 10 friends, most of whom were still abusing it. He had also abused Actified and intravenous amphetamines, but was not currently doing either. He had been in prison for two short periods for stealing to pay for his habit and, although on both occasions he had not undergone serious withdrawal symptoms, he had increased his consumption since then. He had no other psychiatric symptoms. It was calculated that $200 \mathrm{ml}$ of Phensedyl contained $288 \mathrm{mg}$ of ephedrine hydrochloride, $144 \mathrm{mg}$ of promethazine hydrochloride, and $360 \mathrm{mg}$ of codeine phosphate; these amounts were prescribed in tablet form as a daily dose on a reducing regime, and the patient was seen and counselled regularly over a seven-month period. He remained drug-free for a further four months, but his course since then has been one of intermittent Phensedyl abuse and abstinence.

T. M. JELLEY

Royal United Hospital

Combe Park

Bath BA1 3NG

\section{Negative Interaction Between Lithium and ECT}

SIR: We report a woman who developed a mild acute organic brain syndrome with normal serum lithium level, stopped lithium, recovered, and because of continuing depression was given ECT and then developed a severe confusional state.

Case Report: A 57-year-old woman with a history of recurrent unipolar depression was admitted to hospital following the death of her husband. Her father had suffered from depression, but had not sought treatment. He had taken to his bed for 10 years, mainly for depression, but eventually died of senile dementia, Alzheimer's type. There was no other family history of affective disorder. The patient had three children, but none proved to be supportive following the death of her husband.

She was admitted to hospital and treated initially with a variety of antidepressants, including dothiepin, mianserin, and Parnate, but could not tolerate amitriptyline or nortriptyline. Lithium was added to her regime, and although it initially seemed to help her depression she developed severe tremor, ataxia, and a mild confusional state. All medication was therefore stopped. She remained severely depressed and it was decided to give her a course of bilateral ECT.

After the first ECT she complained of mild myalgia and took to her bed, but recovered. ECT appeared to help her depression. However, after the second ECT she became severely confused, rambling, disorientated in time and place, and had a reversal of her sleep rhythm. She then had to be nursed in bed for about a month. She gradually recovered from her confusion and thereafter made a slow recovery. Her depression returned, but using small doses of dothiepin the depression gradually remitted over the next year. She is almost fully recovered from depression, but complains of a poor memory. There are no signs of any dementing illness.

The presence of an ECT-induced confusional state following an episode of lithium toxicity (but with normal serum level) has not been previously reported. While, in retrospect, it might have been more clinically prudent to have delayed ECT, the patient was in a state of very severe depression when this decision was made. Furthermore, we were unaware at the time that there might be any adverse reaction between ECT and lithium.

A few case reports suggest the possibility of such an adverse interaction. Hoenig (1977) reported a patient in whom ECT precipitated a severe encephalopathy, although the patient's serum lithium level was well below the toxic value. Remick (1978) reported a patient who was given ECT while on lithium therapy; after the fourth ECT she developed severe confusion and was incontinent. Six weeks after the resolution of her confusion she was once again treated with ECT, but this time with significant improvement of her depression and no confusion. In a third case (Weiner et al, 1980), lithium carbonate was introduced after a third ECT treatment and the patient developed a severe fluctuating confusional state.

In a retrospective controlled study of 25 patients who had both ECT and lithium, Small et al (1980) found that the lithium/ECT treatment group had more severe memory loss, atypical neurological findings, and a poorer response to ECT. These authors speculated that the patients on lithium might either be a more severely depressed group, or that there might be a true negative interaction between these two common methods of treatment.

Our patient and the patients reported by others suggest the latter, and we are presently investigating the possibility of a true negative interaction between lithium and ECT.

\section{Hayes Grove Priory Hospital \\ Prestons Road \\ Hayes \\ Kent BR2 7AS}

Cane Hill Hospital

Coulsdon

Surrey CR3 $3 Y L$ 


\section{References}

HoENIG, J. \& ChAulK, R. (1977) Delirium associated with lithium therapy and electroconvulsive therapy. Canadian Medical Association Journal, 116, 837-838.

Remick, R. A. (1980) Acute brain syndrome associated with ECT and lithium. Canadian Psychiatric Association Journal, 23, 129-130.

Small, J. G., Kellams, J. J., Milstein, V. \& Small, I. F. (1980) Complications with electroconvulsive treatment combined with lithium. Biological Psychiatry, 15, 103-111.

Weiner, R. D., Whanger, A. D., Erwin, C. W. \& Wilson, W. P. (1980) Prolonged confusional state and EEG seizure activity following concurrent ECT and lithium use. American Journal of Psychiatry, 137, 1452-1453.

\section{Structural Brain Lesions in Functional Psychosis}

SIR: Findings pertinent to cerebral and cerebellar pathology and functional psychosis seem to be limited and contradictory (Hamilton et al, 1983; Wexler, 1980). It has not yet been proven, however, whether there is a significant aetiological relationship between organic pathology and psychiatric illness in general. We present two case reports of functional psychosis: one patient with bipolar affective disorder who had radiological evidence of cerebral and cerebellar atrophy, and another with a schizophrenialike disorder who had cerebellar atrophy. They were both illustrated by $\mathrm{CT}$.

Case Reports: (i) A 49-year-old woman without any personal or family history of neuropsychiatric illness became depressed at the age of 38 , with symptoms of sadness, hopelessness, psychomotor retardation, decreased appetite and sleep, and a serious suicidal attempt. She was admitted to a psychiatric clinic, but within a few days her depression changed to a manic state with increased psychomotor activity, euphoria, loquacity, and delusions of grandeur. She returned to her normal state in about three weeks, but experienced a similar depressive and a manic episode six years later. We examined the patient during her third depressive episode, at which time she was treated with tricyclic antidepressives and lithium. At six-month follow-up, the patient showed a significant deterioration and was noted to have a cerebellar syndrome with symptoms of dysmetria, dysarthria and cerebellar ataxia. Serum lithium level and blood chemistry were normal. EEG revealed nonspecific dysrhythmia, but CT of the brain revealed diffuse cortical atrophy, ventricular dilatation, and cerebellar vermian atrophy. During the following two months her condition gradually merged into a dementia syndrome which was documented by psychological tests. Later she developed a paranoid-hallucinatory and delusional state. Finally, her schizophreniform manifestations improved, but the dementia remained unchanged.

(ii) A 24-year-old female had symptoms of hearing voices and talking and acting as if they were real. She also had fears of being harmed by others, and gradually became withdrawn. Her initial symptoms had occurred at the age of 15 , and she was diagnosed as having an early onset schizophrenic disorder with symptoms of auditory hallucinations, persecutory delusions, delusions of reference, lack of interpersonal relations with flatness of affect, and finally autism. In spite of antipsychotic therapy, the patient's condition worsened and she became severely disabled and dependent on others. Over the past year all psychiatric symptoms disappeared, but she revealed several neurological deficits, including left central facial paresis, left hemiparesis, horizontal nystagmus, ataxia, and dysequilibrium, all of which were preceded by headache, nausea, and vomiting. Currently the patient's neurological findings are the same, and the psychiatric condition is within normal limits. CT was carried out twice, and both times showed cerebellar vermian atrophy.

Heath et al (1979) found CT scan abnormalities in $50 \%$ of 264 patients with functional psychosis, 42 $(32 \%)$ of whom had cerebellar vermian atrophy. Nasrallah et al (1981) reported 43 schizophrenic and 15 manic patients in whom CT findings were consistent with cerebellar atrophy. The main function of the cerebellum is thought to be largely motor, and especially related to co-ordination, tonus, and balance, but some of the clinical and neurophysiological studies revealed new aspects of the cerebellum in relation to autonomic, limbic, and higher cortical systems (Hamilton et al, 1983). Wexler (1980) pointed to right hemispheric dysfunction in manic depressive psychosis and left hemispheric dysfunction in schizophrenic psychosis.

We conclude that there is probably a sub-group of schizophrenic patients in which the symptomatology could be related to structural brain lesions, which is also true for bipolar affective disorder.

SUNAR BIRSÖZ

Akdeniz Üniversitesi Tip Fakültesi

Ali IHSAN Baysal

Psikiatri Anabilim Dal

Antalya

Turkey

\section{References}

Hamilton, G. N., Frick, R. B., Takahashi, T. et al (1983) Psychiatric symptoms and cerebellar pathology. American Journal of Psychiatry, 140, 1322-1326.

Heath, R. G., Franklin, D. E. \& Shraberg, D. (1979) Gross pathology of the cerebellum in patients diagnosed and treated as functional psychiatric disorders. Journal of Nervous and Mental Disease, 167, 585-592.

Nasrallah, H. A., Jacoby, C. G. \& Whitters, M. M. (1981) Cerebellar atrophy in schizophrenia and mania. The Lancet, $i$ 1102.

Weinberger, D. R., Klennman, J. E., Luchins, D. J. et al (1980) Cerebellar pathology in schizophrenia: a controlled post-mortem study. American Journal of Psychiatry, 137, 359-361.

WEXLER, B. E. (1980) Cerebral laterality and psychiatry: a review of the literature. American Journal of Psychiatry, 137, 279-291. 